\title{
Small Choroidal Melanoma Revealed by a Large Extrascleral Extension
}

\author{
Stéphanie Lemaître ${ }^{a, b}$ Mathieu Zmudac Pierre Vincent Jacomet ${ }^{c}$ \\ Christine Lévy-Gabriel $^{a}$ Rémi Dendale ${ }^{d}$ Olivier Berges ${ }^{c}$ Paul Fréneaux $^{a}$ \\ Pascale Mariani $^{a} \quad$ Laurence Desjardins $^{a} \quad$ Olivier Galatoire ${ }^{c} \quad$ Nathalie Cassoux $^{a}$ b \\ ${ }^{a}$ Institut Curie, ${ }^{b}$ Université Paris Descartes, and ${ }^{\mathrm{C}}$ Fondation ophtalmologique Adolphe de Rothschild, Paris, and \\ ${ }^{d}$ Institut Curie, ICPO (Protontherapy Center), Orsay, France
}

\section{Keywords}

Choroidal melanoma · Imaging $\cdot$ Neuritis · Orbital tumor

\begin{abstract}
Aims: Orbital extraocular extension of choroidal melanoma is very rare with small melanomas. We report the case of a patient whose small choroidal melanoma was initially overlooked and was revealed by a large extrascleral extension. Methods: A 48-year-old Caucasian woman presented with sudden total visual loss in the right eye. Multicolor imaging of the fundus showed right optic disc edema and an orange and green lesion near the optic disc. The diagnosis of unilateral optic neuritis was made. Magnetic resonance imaging showed an extraocular mass adjacent to the optic nerve; on ultrasound, this mass was acoustically hollow and a small intraocular component was visible. Results: Choroidal melanoma with a large extrascleral extension was diagnosed (T4eNOM0, stage IIIC according to the AJCC 7th TNM classification, 2010). The size of the extraocular nodule was $13 \times$ $5 \mathrm{~mm}$. Treatment consisted of enucleation followed by adjuvant external beam orbital radiotherapy. Tumor analysis showed a mixed cell type melanoma with monosomy 3 . The patient developed liver metastasis 10 months after local treatment. Conclusion: Extraocular extension of choroidal
\end{abstract}

melanoma can occur with small lesions. Prognosis is generally poor according to AJCC TNM. This case is a reminder that fundus examination may reveal the nature of the mass in some patients with orbital tumors.

(c) 2017 S. Karger AG, Basel

\section{Introduction}

Orbital extraocular extension of choroidal melanoma is very rare with small lesions. We report a rare case of small choroidal melanoma revealed by a compressive optic neuropathy, which was caused by a large extrascleral extension.

\section{Case Report}

A 48-year-old Caucasian woman with no past medical history presented with sudden visual loss in the right eye. On right eye examination, there was no light perception, anterior segment and intraocular pressure were normal. Fundus examination and fluo-

This paper was partially presented at the International Society of Ocular Oncology (ISOO) Annual Meeting in June 2015, Paris, France.

\section{KARGER}

(c) 2017 S. Karger AG, Basel

E-Mail karger@karger.com

www.karger.com/oop
Stéphanie Lemaître

Institut Curie - 26, rue d'Ulm

FR-75248 Paris Cedex 05 (France)

E-Mail stefaniele@ free.fr 


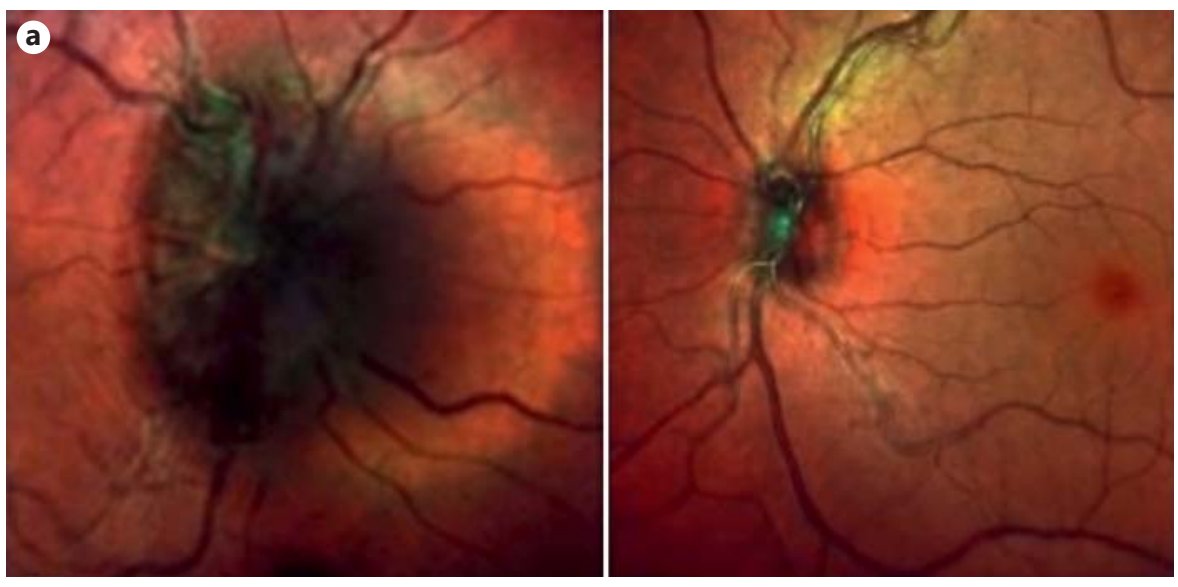

Fig. 1. Initial workup in the diagnosis of unilateral optic neuritis. a Multicolor imaging using the Heidelberg Spectralis showing a right optic disc edema (in brown); a nasal orange and green juxtapapillary lesion can be seen. The optic disc edema appears pigmented due to false colors with the Heidelberg Spectralis imaging device (in reality the only pigmentation seen on fundus examination is in the area of the nasal lesion). Left eye examination is unremarkable. b Fluorescein angiography showing the optic disc edema and the filling of a serous nasal retinal detachment (also seen on the corresponding OCT). Multiple pinpoints are seen in the nasal area adjacent to the optic disc. c Visual fields showing superior nasal quadrantanopia and arcuate scotoma.
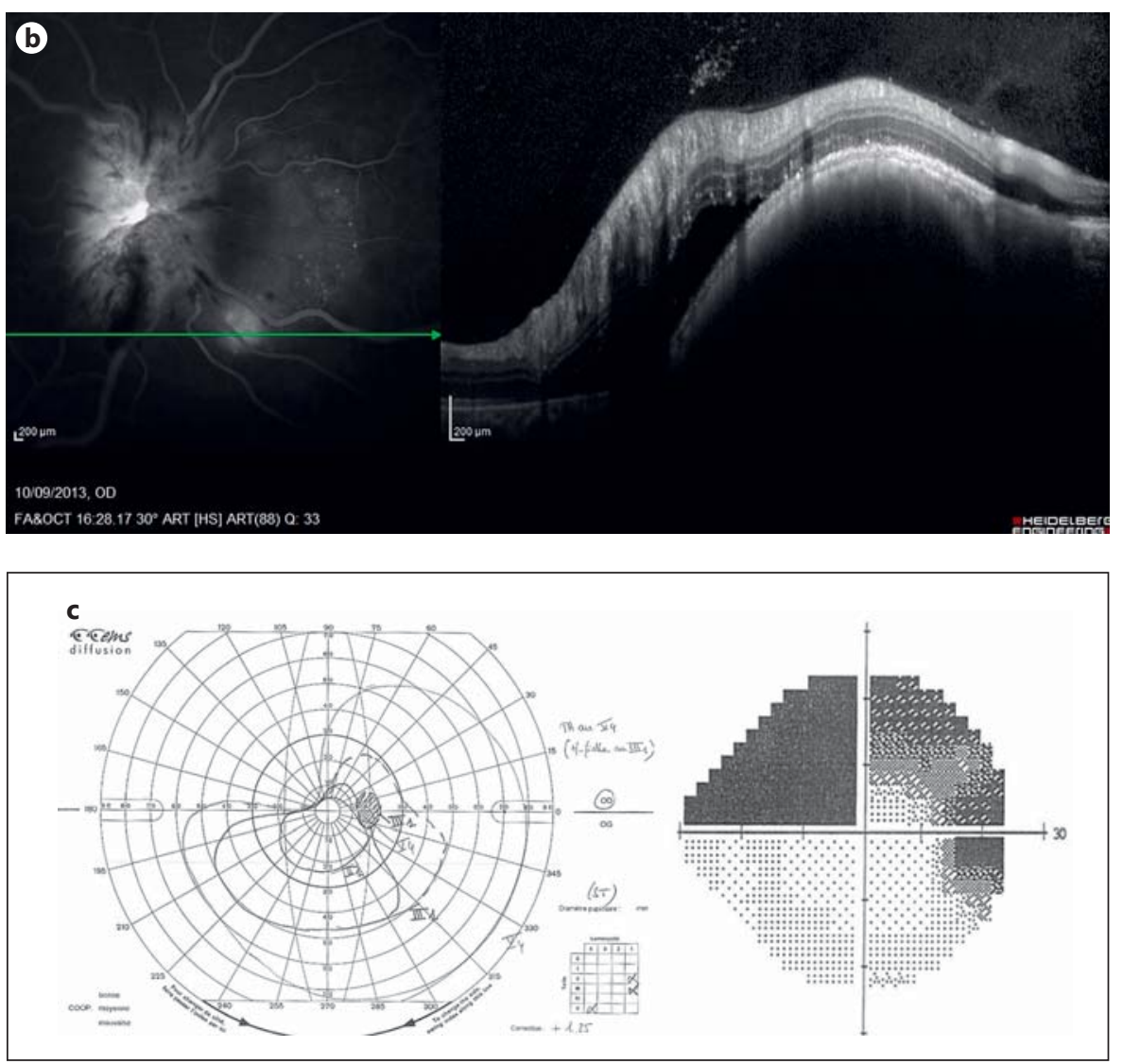

rescein angiography showed an optic disc edema (Fig. 1a, b). Left eye examination was unremarkable. The patient was diagnosed with unilateral optic neuritis and received intravenous and oral corticosteroids which were tapered off. Visual acuity at the end of treatment was 20/20 in the right eye. There was no optic disc edema left on fundus examination and the patient's only complaint was a unilateral scotoma (Fig. 1c). Magnetic resonance imaging (MRI) (performed as part of the optic neuritis investigations) showed a right juxtapapillary extraocular mass which was enhanced after gadolinium injection (Fig. 1d, e). On ocular color
Doppler ultrasound, the extraocular mass was acoustically hollow and vascularized, its dimensions were $7.8 \times 3.8 \mathrm{~mm}$, and a small intraocular component of the mass was noticed (Fig. 2a). Compressive optic neuropathy was diagnosed but systemic evaluation for inflammatory, infectious, and neoplastic disease were unrevealing. Due to the high suspicion for primary ocular malignancy because of the mass on orbital MRI, the patient was seen at the ocular oncology clinic 6 months after the initial visual loss. There had been an increase in the size of the lesion on the ultrasonographic follow-up (Fig. 2a, b), and fundus examination of the right 

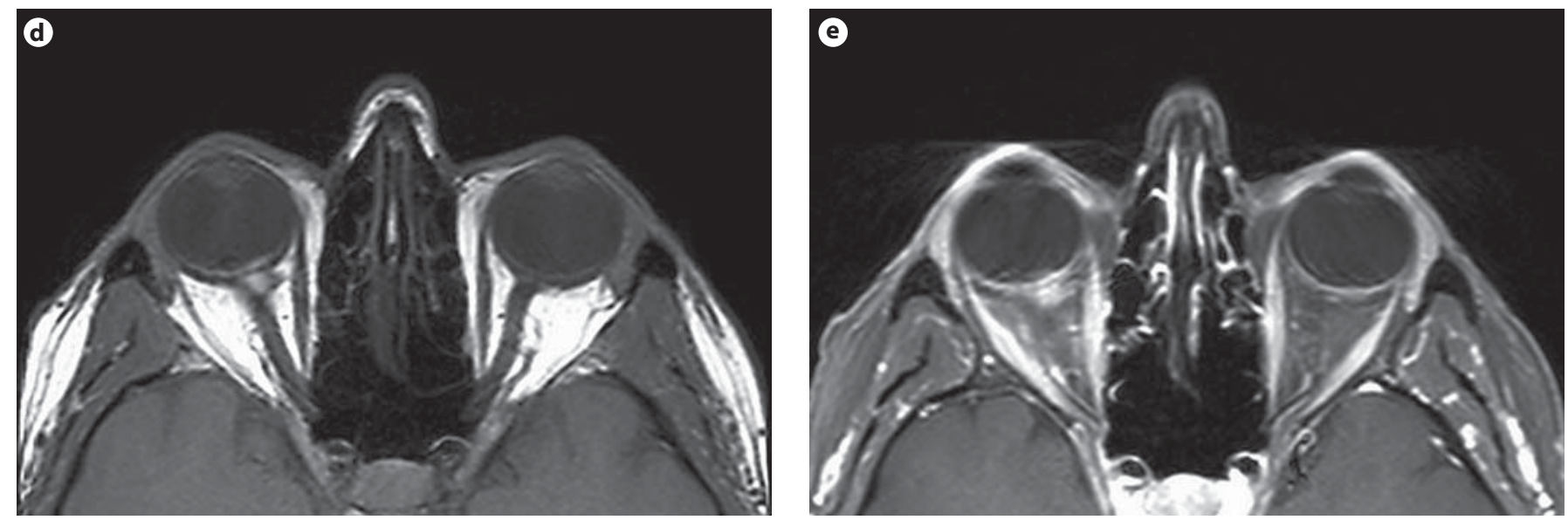

Fig. 1. Initial workup in the diagnosis of unilateral optic neuritis. d, e T1-weighted MRI sequence showing a hyperintense lesion beside the right optic nerve, which is enhanced after gadolinium injection.

eye showed a pigmented juxtapapillary choroidal mass with orange pigment on the surface which was not present on initial examination (Fig. 2c). Visual acuity was still 20/20. The diagnosis of a small juxtapapillary choroidal melanoma with a large extrascleral extension was made. Treatment consisted of enucleation followed by prophylactic external beam radiotherapy of the right orbit at a dose of $54 \mathrm{~Gy}$ in $1.8 \mathrm{~Gy}$ fractions (30 fractions). The irradiation volume covered the entire orbit sparing the eyelids. The radiation technique used was volumetric arc therapy radiation. On histopathological examination, the tumor was a mixed-cell type choroidal melanoma (spindle and epithelioid) T4eN0M0, stage IIIC according to the 2010 AJCC 7th TNM classification [1] (Fig. 2d-h). The size of the extraocular nodule was $13 \times 5 \mathrm{~mm}$. Cytogenetic analysis by array comparative genomic hybridization (arrayCGH) showed monosomy 3 and a normal chromosome 8 . The patient developed liver metastases 10 months after enucleation. MRI was in favor of liver miliary, therefore, an exploratory laparoscopy was performed. Multiple small pigmented liver nodules (at least 8) were found during the procedure, with diameters between 1 and $5 \mathrm{~mm}$ (Fig. 3a, b). Histological analysis confirmed the diagnosis of liver metastasis from choroidal melanoma (Fig. 3c). Array-CGH performed on the metastatic liver nodule showed the same genomic profile as the primary ocular tumor, the only difference being an additional loss of chromosomes 15 and 18. Metastatic disease progressed under chemotherapy (intravenous fotemustine) with an increase in the size of liver nodules on MRI scans and the appearance of new metastases located in the vertebrae, the sternum, and the left breast. Death occurred 7 months after the diagnosis of the metastases.

\section{Discussion}

Choroidal melanoma is the most common primary intraocular malignancy in adults. It usually presents at about 60 years of age. The diagnosis is made based on the clinical and ultrasonographic features of the choroidal mass. Documented tumor growth is a highly suggestive criterion for a malignant tumor. In most cases, choroidal melanomas are confined within the scleral envelope. Extraocular extension is present at the time of diagnosis in only $3-5.8 \%$ of the patients with uveal melanoma [2-4]. It is more common in medium or large size tumors and in tumors located in the ciliary body, at the site of vortex veins and in the juxtapapillary area. In a series of patients treated by primary enucleation (therefore having medium to large size choroidal melanomas), $14.6 \%$ had extraocular tumor extension [5]. In most cases, the extraocular extension had occurred through the natural channels of the sclera (aqueous channels, ciliary arteries, vortex veins, and ciliary nerves). Extension along the optic nerve is less frequent as it represents only $0.8 \%$ of the cases [5]. According to Lindegaard et al. $[6,7]$, optic nerve invasion may occur in a neurotropic subtype of uveal melanomas. It has been suggested that in some cases of small tumors with large extraocular extension, tumor cells may have originated from melanocytes which are normally present in the emissary channels along arteries, veins, and nerves [8]. Erosion of the sclera is a very unusual route of extraocular extension which may occur after intraocular surgery [5].

Cytogenetic analysis has become important in choroidal melanoma in order to estimate the risk for metastatic disease and therefore patient prognosis. Monosomy 3 and chromosome 8q gain in the primary tumor have been associated with an increased risk for metastasis [9]. Extraocular spread has also been showed to be correlated with monosomy 3 and with metastatic death [5], but it 
Fig. 2. Diagnosis of a small choroidal melanoma with a large extrascleral extension. a The extraocular mass is acoustically hollow and vascularized on ocular color Doppler ultrasound. A small intraocular component can be seen. b Tumor growth of the intraocular tumor was documented on ultrasonographic follow-up. c Color fundus photography showing a pigmented choroidal mass with orange pigment on the surface. The diameter of the lesion is larger compared with the fluorescein angiography in Figure 1 b. d A pigmented nodule adjacent to the optic nerve can be seen on the enucleation specimen.
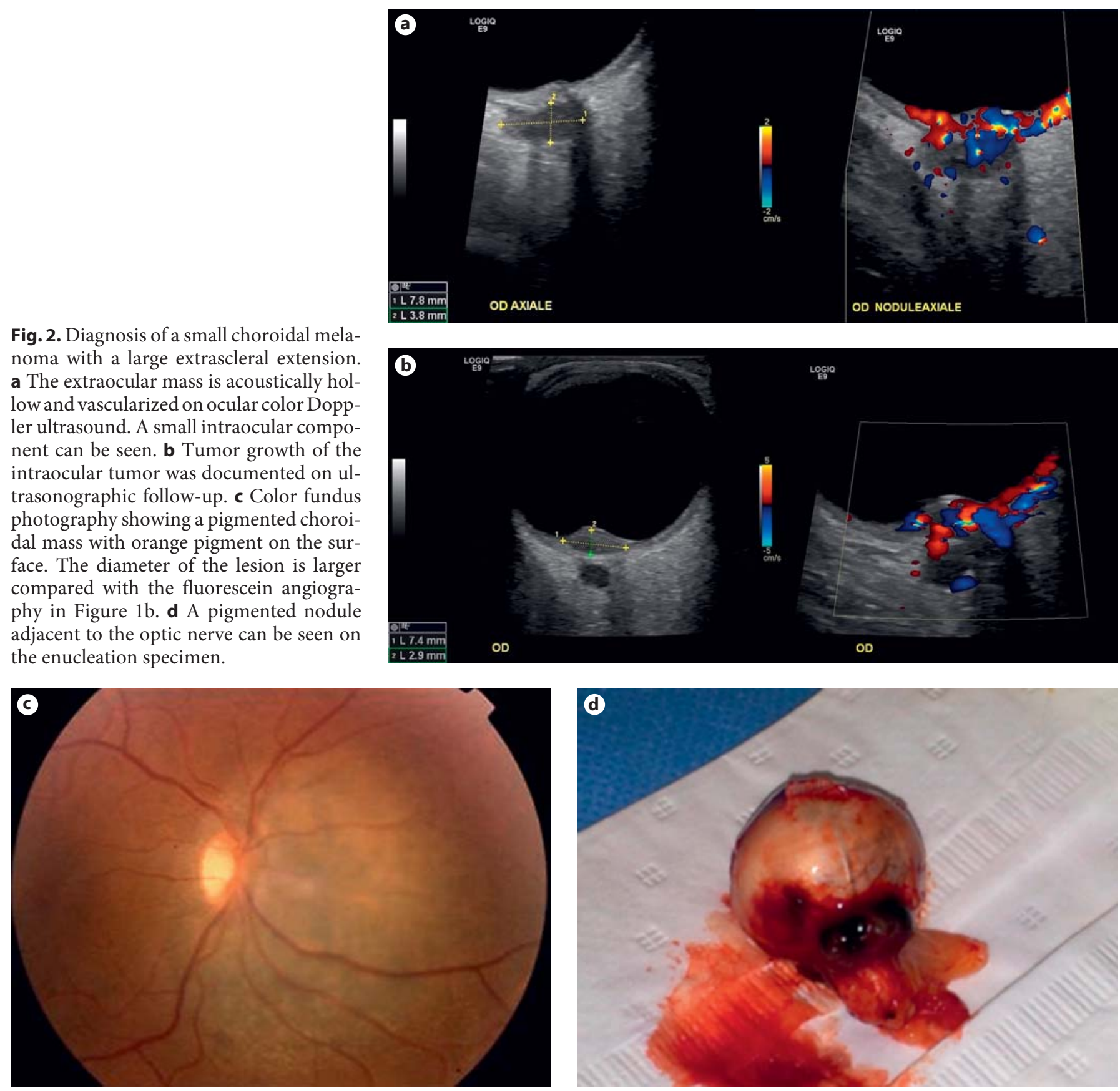

can occur with tumors which do not harbor the cytogenetic features associated with an increased risk for metastases. For example, in the case of a small choroidal melanoma with a large extrascleral extension, cytogenetic analysis showed no monosomy 3 or $8 \mathrm{q}$ gain [10]. In our patient, chromosome 3 monosomy was found on the primary tumor and metastatic disease occurred 10 months after enucleation.

We report on an unusual case of small choroidal melanoma revealed by a misdiagnosed compressive optic neuropathy due to a large extraocular extension. The tumor was initially overlooked because the initial diagnosis was optic neuritis, and systemic evaluation focused on potential inflammatory and infectious causes, hence the diagnostic delay of 6 months. A case similar to ours with sudden visual loss due to optic disc swelling has been re- 

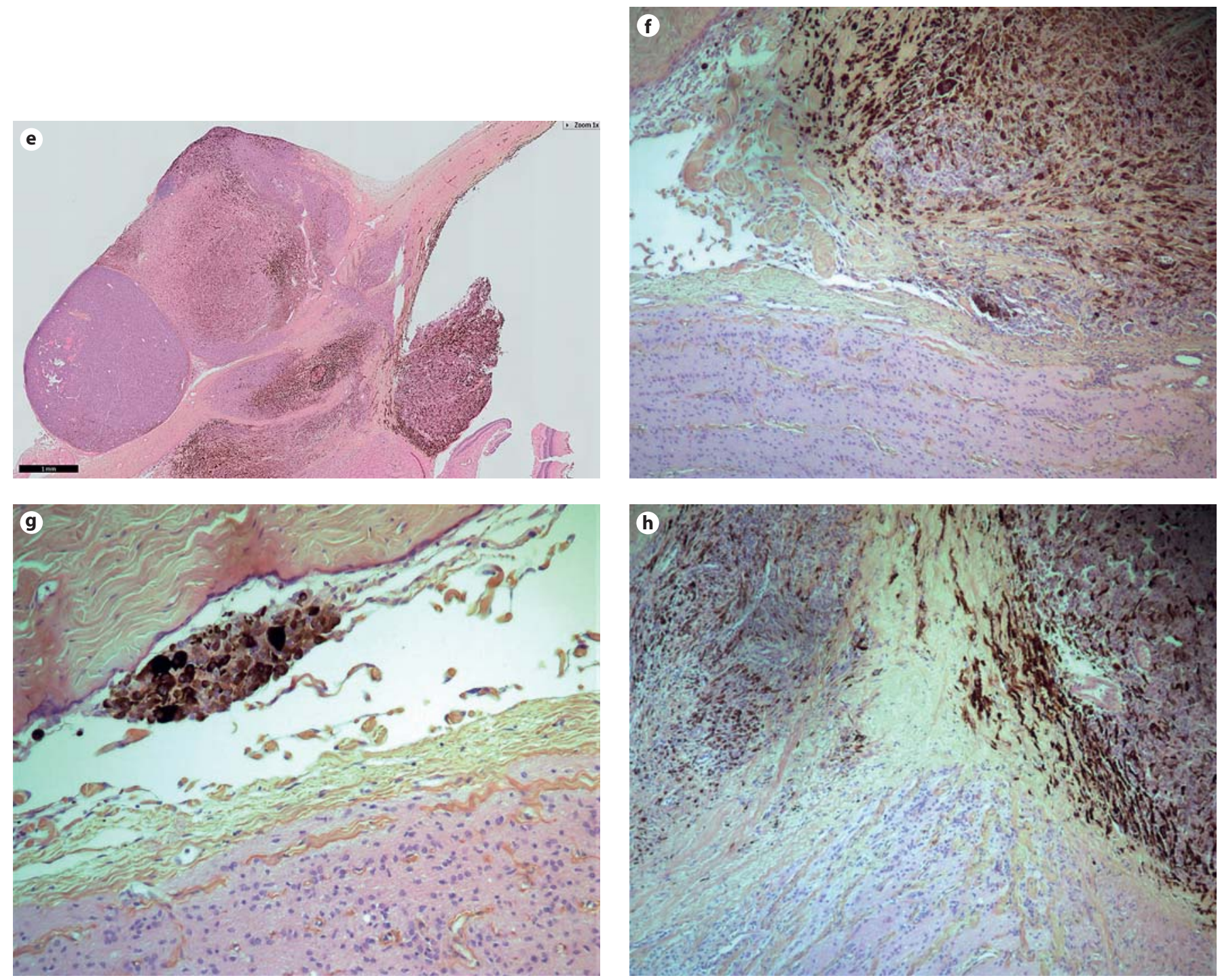

Fig. 2. Diagnosis of a small choroidal melanoma with a large extrascleral extension. e Histology section through the optic nerve showing the extraocular extension and the small intraocular choroidal melanoma (hematoxylin and eosin staining). There is a dual extraocular growth pattern: peripapillary and into the optic nerve

sheaths. f Tumor invasion of the meningeal sheaths (periphery of the optic nerve). $\mathbf{g}$ Small tumor nodule in the meningeal sheaths a little away from the main meningeal tumor invasion. $\mathbf{h}$ Scleral and extrascleral tumor invasion (periphery of the optic nerve, outside the meningeal sheaths).

ported in 1999 [11]. In our patient, the intraocular tumor was initially overlooked because of the large extraocular extension; therefore, it is important that all patients with orbital tumors undergo fundus examination which may in some cases reveal the nature of the orbital mass. The intraocular tumor may also have been overlooked because of false colors on the initial multicolor imaging of the fundus. Indeed, an orange and green juxtapapillary lesion was already present on the initial image of the optic disc but it was not interpreted as a possible choroidal tu-

mor (Fig. 1a). Fluorescein angiography showed pinpoints in this area, but no relation was established between the orange lesion on multicolor imaging and the pinpoints, possibly because clinicians focused on the optic disc edema and the large extraocular mass. False colors make multicolor imaging more operator dependent than color fundus photographs and they may be a cause of misdiagnosis $[12,13]$.

The choice of the treatment modality for choroidal melanoma depends on the size and location of the tumor. 

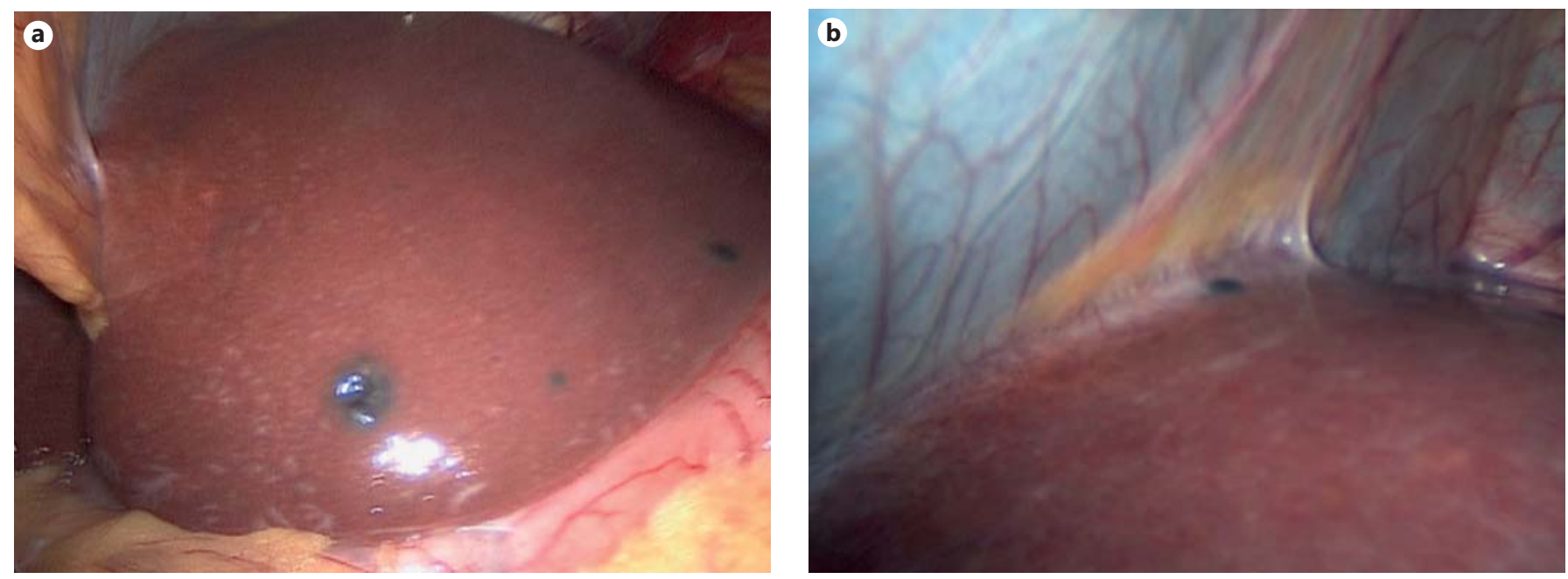

Fig. 3. Progression to metastatic disease. a, b Photographs of the liver taken during the exploratory laparoscopy showing multiple pigmented nodules. c Histology section of 1 of the liver nodules showing multiple melanocytes within the liver tissue.

Primary enucleation is indicated for large tumors (largest basal diameter $>16 \mathrm{~mm}$ and/or tumor thickness $>10 \mathrm{~mm}$ ), whereas globe-sparing treatments are possible for smaller tumors [3]. There is no consensus on the treatment of small choroidal melanomas with a large extraocular extension. Mutilating therapies have been described in the past, like enucleation with partial orbital exenteration through a lateral orbitotomy, followed by postoperative radiation therapy [8]. Currently, if the extraocular nodule is small and anterior, a globe-sparing treatment based on irradiation can be performed in most cases, with no adverse effect on survival and recurrence rates [3]. If the extrascleral nodule is posterior, enucleation followed by adjuvant orbital radiation therapy can be performed in order to reduce the risk of orbital recurrence [14]. A con-

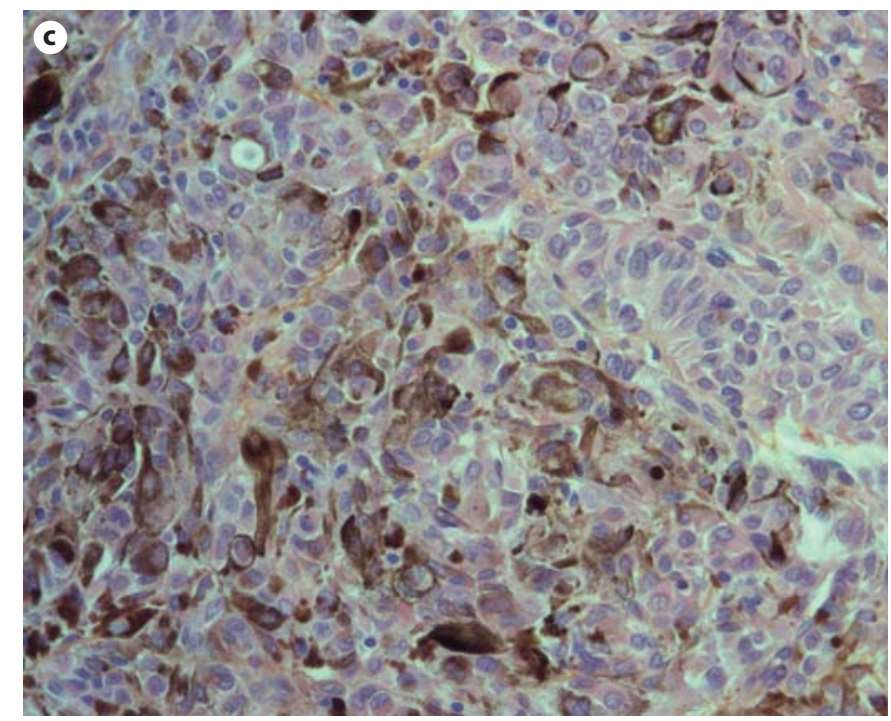

servative therapeutic approach has been decided in the case of a small choroidal melanoma with a large posterior extrascleral extension. The approach consisted of the excision of the extraocular nodule followed by proton beam irradiation of the intraocular tumor and orbital tissues at the site of the excised nodule [10]. In our case, there was a large posterior nodule adjacent to the optic nerve, making any attempt to remove the nodule difficult. Thus, treatment consisted of enucleation followed by adjuvant orbital irradiation. After the removal of the globe, there was no visible residual pigment in the orbit. Histological analysis of the enucleated globe showed a dual extraocular growth pattern: in part, peripapillary (along posterior ciliary arteries or nerves) but also into the optic nerve sheaths. The presence of several nodules between the du- 
ral sac and the nerve justify the primary enucleation because it would not have been possible to resect that part of the tumor without damaging the nerve. On histology, the largest nodules are likely to cause the disk edema.

Primary orbital radiotherapy without performing the enucleation was not a treatment option. If the eye had been left in place, higher radiation doses would have been necessary to control the melanoma. There would have been too many ocular complications at such high doses (and secondary enucleation would have been inevitable). Primary enucleation allowed the entire removal of the entire tumor and was therefore the safest option when followed by adjuvant orbital external beam radiotherapy.

In our patient, both the occurrence of an extrascleral extension and the rapidly evolving metastases are likely related to the aggressive cytogenetic features of the tumor. According to the TNM classification of ciliary body and choroidal melanomas, the category of anatomic extent of the tumor was T4eNOM0 because of the extraocular extension with a diameter larger than $5 \mathrm{~mm}$ (stage IIIC) $[1,4]$. Group IIIC is the highest category, with only $26 \%$ survival at 5 years $(10 \%$ at 10 years and $0 \%$ at 15 years). This fits with the early clinical metastases in the case reported here.

\section{Statement of Ethics}

This study adhered to the Declaration of Helsinki.

\section{Disclosure Statement}

The authors declare that there are no conflicts of interest.

\section{References}

1 Edge SB, Byrd DR, Compton CC, Fritz AG, Greene FL, Trotti A (eds): Uveal Melanoma. AJCC Cancer Staging Manual, ed 7. New York/London, Springer, 2009, pp 547-559.

2 Shields JA, Shields CL: Massive orbital extension of posterior uveal melanomas. Ophthal Plast Reconstr Surg 1991;7:238-251.

3 Bellmann C, Lumbroso-Le Rouic L, Levy C, Plancher C, Dendale R, Sastre-Garau X, et al: Uveal melanoma: management and outcome of patients with extraocular spread. Br J Ophthalmol 2010;94:569-574.

4 Kujala E, Damato B, Coupland SE, Desjardins L, Bechrakis NE, Grange J-D, et al: Staging of ciliary body and choroidal melanomas based on anatomic extent. J Clin Oncol 2013;31: 2825-2831.

5 Coupland SE, Campbell I, Damato B: Routes of extraocular extension of uveal melanoma: risk factors and influence on survival probability. Ophthalmology 2008;115:1778-1785.
6 Lindegaard J, Isager P, Prause JU, Heegaard S: Optic nerve invasion of uveal melanoma: clinical characteristics and metastatic pattern. Invest Ophthalmol Vis Sci 2006;47:3268-3275.

7 Lindegaard J, Isager P, Prause JU, Heegaard S: Optic nerve invasion of uveal melanoma. APMIS 2007;115:1-16.

8 Sambuelli R, Luna JD, Reviglio VE, Aoki A, Juarez CP: Small choroidal melanoma with massive extraocular extension: invasion through posterior scleral emissary channels. Int Ophthalmol 2001;24:213-218.

9 Cassoux N, Rodrigues MJ, Plancher C, Asselain B, Levy-Gabriel C, Lumbroso-Le Rouic L, et al: Genome-wide profiling is a clinically relevant and affordable prognostic test in posterior uveal melanoma. Br J Ophthalmol 2014; 98:769-774.

10 Papadopoulou D, Moulin AP, Zografos L, Schalenbourg A: How would you manage this small melanocytic choroidal tumour? Small choroidal melanoma hiding a large extrascleral extension. Br J Ophthalmol 2012;96: $1530,1539-1540$
11 Shields CL, Santos MC, Shields JA, Singh AD, Eagle RC: Extraocular extension of unrecognized choroidal melanoma simulating a primary optic nerve tumor: report of two cases. Ophthalmology 1999;106:1349-1352.

12 Schalenbourg A, Zografos L: Pitfalls in colour photography of choroidal tumours. Eye (Lond) 2013;27:224-229.

13 Nezgoda JT, Camacho N, Bartsch D-U, Currie G, Freeman WR: Multicolor imaging compared with color fundus photography for retinal, choroidal and optic nerve pathology. Invest Ophthalmol Vis Sci 2014;55:248.

14 Finger PT, Tena LB, Semenova E, Aridgides P, Choi WH: Extrascleral extension of choroidal melanoma: post-enucleation high-doserate interstitial brachytherapy of the orbit. Brachytherapy 2014;13:275-280. 